\title{
Maternal folic acid supplement use in early pregnancy and child behavioural problems: The Generation R Study
}

\author{
Sabine J. Roza ${ }^{1,2}$, Tamara van Batenburg-Eddes ${ }^{1,2}$, Eric A. P. Steegers ${ }^{3}$, Vincent W. V. Jaddoe ${ }^{1,4,5}$, \\ Johan P. Mackenbach ${ }^{6}$, Albert Hofman ${ }^{5}$, Frank C. Verhulst ${ }^{2}$ and Henning Tiemeier ${ }^{2,5 *}$ \\ ${ }^{1}$ The Generation R Study Group, Erasmus MC University Medical Center Rotterdam, Dr. Molewaterplein 50, 3015 GE Rotterdam, \\ The Netherlands \\ ${ }^{2}$ Department of Child and Adolescent Psychiatry, Erasmus MC University Medical Center Rotterdam, Dr. Molewaterplein 50, 3015 \\ GE Rotterdam, The Netherlands \\ ${ }^{3}$ Department of Obstetrics and Gynaecology, Erasmus MC University Medical Center Rotterdam, Dr. Molewaterplein 50, 3015 GE \\ Rotterdam, The Netherlands \\ ${ }^{4}$ Department of Paediatrics, Erasmus MC University Medical Center Rotterdam, Dr. Molewaterplein 50, 3015 GE Rotterdam, \\ The Netherlands \\ ${ }^{5}$ Department of Epidemiology, Erasmus MC University Medical Center Rotterdam, Dr. Molewaterplein 50, 3015 GE Rotterdam, \\ The Netherlands \\ ${ }^{6}$ Department of Public Health, Erasmus MC University Medical Center Rotterdam, Dr. Molewaterplein 50, 3015 GE Rotterdam, \\ The Netherlands
}

(Received 4 February 2009 - Revised 10 August 2009 - Accepted 11 August 2009 - First published online 22 September 2009)

\begin{abstract}
Folate deficiency during embryogenesis is an established risk factor for neural tube defects in the fetus. An adequate folate nutritional status is also important for normal fetal growth and brain development. The aim of the present research was to study the association between folic acid use of the mother during pregnancy and child behavioural development. Within a population-based cohort, we prospectively assessed folic acid supplement use during the first trimester by questionnaire. Child behavioural and emotional problems were assessed with the Child Behaviour Checklist at the age of 18 months in 4214 toddlers. Results showed that children of mothers who did not use folic acid supplements in the first trimester had a higher risk of total problems (OR 1.44; $95 \%$ CI 1.12, 1.86). Folic acid supplement use protected both from internalising (OR of no supplement use 1.65; $95 \%$ CI 1.24, 2.19) and externalising problems (OR 1.45; $95 \%$ CI 1.17, 1.80), even when adjusted for maternal characteristics. Birth weight and size of the fetal head did not mediate the association between folic acid use and child behaviour. In conclusion, inadequate use of folic acid supplements during early pregnancy may be associated with a higher risk of behavioural problems in the offspring. Folic acid supplementation in early pregnancy, aimed to prevent neural tube defects, may also reduce mental health problems in children.
\end{abstract}

Folic acid supplements: Pregnancy: Infant behaviour: Epidemiology

Folic acid, an essential micronutrient, is involved in the synthesis of DNA, RNA and proteins ${ }^{(1)}$. A deficiency in folate during pregnancy is a well-known risk factor for neural tube defects ${ }^{(2,3)}$. Moreover, an adequate folate status is important for normal fetal growth ${ }^{(4)}$. Animal studies have established essential roles for folates in the development of the central nervous system as well. Folates influence neuronal and glial growth and proliferation, and are indirectly involved in the synthesis of neurotransmitters, such as dopamine, noradrenaline, adrenaline and serotonin ${ }^{(5,6)}$.

Information on the association between prenatal folate status and human neurodevelopment after closure of the neural tube is scarce and inconclusive. Following the reported coincidence of an increased risk of schizophrenia with an increased risk of neural tube defects in mothers exposed to severe famine during the preconceptional period, it has been hypothesised that maternal low folate is a risk factor for developing schizophrenia ${ }^{(7)}$. Brown et al. found an association between maternal hyperhomocysteinaemia, which is inversely related to folate, in the third trimester of pregnancy, and the risk of adult schizophrenia $^{(8)}$. Several inborn errors of folate mechanism give rise to mental retardation and autistic symptoms ${ }^{(9,10)}$. On the other hand, Tamura et al. found no differences in neuropsychological test scores between children exposed to low and high folates in pregnancy ${ }^{(11)}$. Whether maternal folate deficiencies are related to behavioural problems in the offspring is yet unclear.

Abbreviation: CBCL, Child Behaviour Checklist.

* Corresponding author: Dr Henning Tiemeier, fax +31 10 7044657, email h.tiemeier@erasmusmc.nl 
The present study examines the association between folic acid use during the first trimester of pregnancy and child behavioural and emotional problems. We hypothesised that inadequate folic acid supplement use during embryogenesis was related to a higher risk of behavioural problems in the offspring.

\section{Methods}

\section{Setting}

The present study was conducted within the Generation $\mathrm{R}$ Study, a population-based cohort in Rotterdam, The Netherlands ${ }^{(12)}$. Enrolment was aimed in early pregnancy. All children were born between April 2002 and January 2006 and form a prenatally enrolled birth cohort that is currently being followed until young adulthood. The study was conducted in accordance with the guidelines proposed in the World Medical Association Declaration of Helsinki and has been approved by the Medical Ethics Committee of the Erasmus Medical Center, Rotterdam. After complete description of the study to the adult subjects, written informed consent was obtained.

\section{Study population}

In total, 7654 live born children and their mothers, who were prenatally included in Generation $\mathrm{R}$, were approached for postnatal consent. Of these, mothers without information on folic acid supplement use in early pregnancy were excluded ( $n 1139 ; 14.9 \%$ ). Of the children, twenty-four died in the first few months after birth. The remaining 6491 children were eligible for the present study. Mothers of 788 children refused full consent for postnatal participation. Another 1489 mothers did not complete the 18-month questionnaire. Information on child behavioural problems at age 18 months was available in 4214 toddlers (64.9\% of 6491 toddlers). Some mothers participated with two children ( $n$ 268), and another four mothers participated with three children. Since results did not differ after random exclusion of one or two of these siblings, they were included in the analyses.

\section{Maternal folic acid use}

Information on folic acid use as a single or multivitamin preparation in the first trimester was obtained by questionnaire in early pregnancy (gestational age $<18$ weeks). Pregnant women were asked whether they used folic acid supplements and when supplementation was started (before pregnancy, as soon as pregnancy was known, later in pregnancy). Since results did not differ between women who started folic acid supplement use preconceptionally and women who started within the first 10 weeks of pregnancy, we categorised folic acid supplement use into 'preconceptional start or start during the first 10 weeks of pregnancy' and 'no use during embryogenesis'.

Self-reported folic acid supplement use was validated by serum folate levels in a small subsample of the present study (n 272). Blood samples were collected between 7 and 12 weeks of gestation. Within mothers who reported using folic acid supplements ( $n$ 204; $75 \%$ ), the median of serum folate was $23.5 \quad(95 \%$ CI $4.3,45 \cdot 3) \mathrm{nmol} / \mathrm{l}$, whereas the median serum folate level within mothers who did not use folic acid supplements ( $n 68 ; 25 \%)$ was $11.1(95 \%$ CI $4.7,29.6) \mathrm{nmol} / \mathrm{l}$. The difference in distribution function (Mann-Whitney test) is statistically significant $(P<0 \cdot 001)$.

\section{Child behavioural and emotional problems}

The Child Behaviour Checklist for toddlers (CBCL/1 $\left.\frac{1}{2}-5\right)$ is a self-administered questionnaire and was used to obtain standardised parent reports of children's problem behaviours. The CBCL/ $1 \frac{1}{2}-5$ contains ninety-nine problem items, which are scored on seven empirically based syndromes that were derived by factor analyses: emotionally reactive, anxious/ depressed, somatic complaints, withdrawn, sleep problems, attention problems, and aggressive behaviour. The broadband scale 'internalising' is the sum score of items in the first four syndrome scales, whereas 'externalising' is the sum score of attention problems and aggressive behaviour. 'Total problems' is the sum score of all ninety-nine problem items. Each item is scored $0=$ not true, $1=$ somewhat or sometimes true, and $2=$ very true or often true, based on the preceding 2 months. Good reliability and validity have been reported for the $\mathrm{CBCL}^{(13)}$. We used the borderline cut-off scores (83rd percentile for the broadband scales and 93rd percentile for the syndrome scales of a Dutch norm group ${ }^{(14)}$ ) to classify children as having behavioural problems in the borderline or clinical range.

\section{Covariates}

Date of birth, birth weight, birth order and sex of the infant were obtained from midwife and hospital registries at birth. We established gestational age by using the fetal ultrasound examinations within the Generation R Study. Head circumference, biparietal diameter, transcerebellar diameter and atrial width of the lateral ventricle were assessed by fetal ultrasound in late pregnancy (median gestational age 30 weeks, $95 \%$ CI 28,33 weeks) using standardised ultrasound protocols. Information about parental age, educational level, national origin, maternal psychopathology and pregnancy planning was obtained by questionnaire. We adjusted our associations for both maternal and paternal age, since several studies showed associations between parental age and neurodevelopmental outcome $^{(15,16)}$. The highest completed education (primary school, secondary school, and higher education) was decided on the educational level of the mother. The pregnant woman was of non-Dutch origin if one of her parents was born abroad. If both parents of the participating mother were born abroad, we used the country of birth of the mother of the mother to classify the ethnic background. We classified national origin into five categories: (a) Dutch; (b) other Western; (c) Turkish or Moroccan; (d) Surinamese or Antillean; (e) other non-Western. To assess maternal and paternal psychopathology in mid-pregnancy (gestational age 18-25 weeks), we used the Brief Symptom Inventory ${ }^{(17,18)}$. The score on the Global Severity Index was standardised and used as a continuous variable. Maternal smoking and maternal alcohol use were assessed at three time points during pregnancy (early pregnancy, mid-pregnancy and late pregnancy) and categorised into 'yes, during pregnancy' and 'no use 
during pregnancy'. We measured weight and height of the pregnant women three times in pregnancy during visits at the research centre. BMI was calculated by dividing weight by squared height. Breast-feeding was assessed at 2, 6 and 12 months of age. We categorised breast-feeding into 'stopped before 3 months of age' and 'continued breast-feeding after 3 months of age'.

\section{Statistical analyses}

Differences in baseline characteristics between mothers who used and did not use folic acid supplements were compared with the $\chi^{2}$ statistic for categorical variables, independent $t$ tests for continuous normally distributed variables and Mann-Whitney $U$ tests for continuous non-normally distributed variables. Successive logistic regression models are presented to show whether the association between maternal folic acid use and behavioural problems holds up when potential confounders are controlled. Folic acid supplement use was the reference category in these analyses, and the OR represent a higher risk of behavioural problems in children of mothers who did not use folic acid supplements. Confounders were included in the final models if the effect estimate of folic acid use changed meaningfully (defined as more than $5 \%$ ). Interaction terms of folic acid use with all confounding variables were tested and included at $\alpha=0 \cdot 15$. Measures of association are presented with their $95 \%$ CI. All quoted significance levels are two-sided $P$ values. Statistical analyses were carried out using SPSS (version 11.0 for Windows; SPSS Inc., Chicago, IL, USA).

\section{Response analyses}

Analyses of missing data showed that children without information on behaviour weighed on average 110 (95\% CI 81, 139) g lower at birth $(t=7 \cdot 4 ; P<0.001)$, and had shorter gestation (median 40.0 (95\% CI 34.9, 42.3) weeks $v .40 .1(95 \%$ CI $35.6,42.4)$ weeks; $P<0.001)$ than children with behavioural data. Their mothers were on average 3.0 (95\% CI $2 \cdot 8,3 \cdot 3)$ years younger $(t=22.4 ; P<0.001)$, lower educated $\left(19.9 \%\right.$ primary level v. $5.7 \% ; \chi^{2}=714$ (df 2); $\left.P<0.001\right)$, and less often of Dutch origin (29.6 v. $63.3 \% ; \chi^{2}=858$ (df 4); $P<0.001$ ) than mothers of children with behavioural data. They less often used folic acid supplements during the first trimester $\left(44.9\right.$ v. $\left.72.8 \% ; \chi^{2}=526(\mathrm{df} 1) ; P<0.001\right)$.

\section{Results}

A description of the participating women and their children is presented in Table 1 . Several child and maternal characteristics differed widely between mothers who used folic acid during embryogenesis and mothers who did not. A total of 3067 mothers used $400 \mu \mathrm{g}$ folic acid during embryogenesis. Of these, $1886(61.5 \%)$ mothers started supplement use at least 4 weeks before conception, whereas the other 1181 mothers started use as soon as pregnancy was known, within the first 10 weeks of gestation. In addition, 1274 mothers reported multivitamin preparation use at 12 weeks of gestation. Mothers who used folic acid were more often of Dutch origin, higher educated, were older, and had lower psychopathology scores than mothers who did not used folic acid (Table 1). Folic acid supplementation during pregnancy was highly related to lifestyle factors as well, which can be seen in the differences in smoking, alcohol use and maternal BMI (see also Timmermans et al. ${ }^{(19)}$ ). The children of mothers who used folic acid had higher birth weights, larger head circumferences and larger ventricular sizes in late pregnancy than children of mothers who did not use folic acid (Table 1). Although the medians and $95 \%$ CI of gestational age at birth between folic acid users and non-users seem very similar, the difference in distribution function is statistically significant. Children of mothers who did not use folic acid supplements were relatively more born during week 38 and $39(12.3+25.6 \%$ in non-users $v .10 .4$ and $21.6 \%$ in folic acid users), whereas children of mothers who used folic acid supplementation had a higher chance of being born during week 41 of gestation $(20.6 \%$ in folic acid users v. $14.8 \%$ in non-users).

Table 2 shows the association between the possible confounding variables and behavioural problems at the age of 18 months. Many of the considered covariates, such as age and sex of the child, birth weight, national origin, maternal educational level, smoking and alcohol use, maternal psychopathology and parental age, were significantly related to behavioural problems.

Table 3 shows the association between maternal folic acid use during the first trimester and the CBCL total problems score at age 18 months. Model 1 of Table 2 was only adjusted for age and sex of the child. The OR for behavioural problems in children of mothers who did not use folic acid supplements during embryogenesis was 2.8 (95\% CI 2.2, 3.4) compared with children of mothers who used folic acid supplements. Model 2 introduces several confounding variables in the association of folic acid supplementation and child behavioural problems. The higher risk of behavioural problems in children of mothers who did not use folic acid supplements, albeit reduced to $1.4(95 \%$ CI $1.1,1.9)$, remained statistically significant after adjustment for maternal age, educational level, national origin and psychopathology. Other parental characteristics, such as pregnancy planning, maternal smoking during pregnancy, maternal alcohol use during pregnancy, maternal BMI, paternal age and breast-feeding, did not further confound the effect of folic acid use on behavioural problems. Models 3 and 4 (see Table 3) show whether the association between folic acid use and offspring behaviour was mediated by fetal growth. Birth weight and head circumference were not related to behavioural outcome after adjustment for maternal characteristics and therefore did not mediate the association between folic acid use and child behaviour. Gestational age at birth was related to behavioural outcome (OR 0.9; $95 \%$ CI $0.9,1.0$ ), but did not change the effect estimate of folic acid use on behavioural outcome. Similarly, transcerebellar diameter and atrial width of the lateral ventricle during late pregnancy did not explain the association between folic acid use and child behavioural problems (OR after adjustment for these factors $1.4(95 \%$ CI $1.1,1.9 ; P=0.004))$. None of the interaction terms between folic acid use and the explanatory variables was significant at $\alpha=0 \cdot 15$.

To avoid major effects of misclassification of the determinant, we also conducted the following analyses. First, within a subsample of 272 mothers, we used actual serum folate level to classify users and non-users at a cut-off value of 
Table 1. Subject characteristics in offspring of mothers using folic acid supplementation during embryogenesis (Mean values and standard deviations, medians and $95 \%$ confidence intervals or percentages)

\begin{tabular}{|c|c|c|c|c|c|}
\hline & \multicolumn{2}{|c|}{$\begin{array}{l}\text { Folic acid use } \\
\qquad(n \text { 3067) }\end{array}$} & \multicolumn{2}{|c|}{$\begin{array}{l}\text { No folic acid use } \\
\qquad(n 1147)\end{array}$} & \multirow[b]{2}{*}{$P^{\star}$} \\
\hline & Mean & SD & Mean & SD & \\
\hline Male (\%) & \multicolumn{2}{|c|}{$48 \cdot 7$} & \multicolumn{2}{|c|}{50.5} & 0.30 \\
\hline Gestational age at birth (weeks) & \multirow{2}{*}{\multicolumn{2}{|c|}{$40 \cdot 1$}} & & & $<0.001$ \\
\hline Median & & & \multicolumn{2}{|c|}{$40 \cdot 0$} & \\
\hline $95 \% \mathrm{Cl}$ & \multicolumn{2}{|c|}{$35 \cdot 6,42 \cdot 4$} & \multicolumn{2}{|c|}{$36 \cdot 0,42 \cdot 3$} & \\
\hline Preterm birth < 37 weeks (\%) & \multicolumn{2}{|c|}{$5 \cdot 1$} & \multicolumn{2}{|c|}{4.4} & 0.42 \\
\hline Birth weight $(\mathrm{g})$ & 3493 & 545 & 3384 & 546 & $<0.001$ \\
\hline Low birth weight $<2500 \mathrm{~g}(\%)$ & \multicolumn{2}{|c|}{3.6} & \multicolumn{2}{|c|}{5.6} & 0.004 \\
\hline Head circumference in late pregnancy $(\mathrm{mm})$ & $286 \cdot 3$ & $12 \cdot 0$ & 284.9 & $12 \cdot 0$ & 0.001 \\
\hline Transcerebellar diameter in late pregnancy $(\mathrm{mm})$ & 37.9 & 2.4 & 37.8 & 2.5 & 0.77 \\
\hline Atrial width of lateral ventricle in late pregnancy $(\mathrm{mm})$ & $5 \cdot 0$ & $1 \cdot 7$ & 4.8 & $1 \cdot 7$ & 0.001 \\
\hline National origin $(\%)$ & & & & & $<0.001$ \\
\hline Dutch & \multicolumn{2}{|c|}{$71 \cdot 8$} & \multicolumn{2}{|c|}{$40 \cdot 1$} & \\
\hline Other Western & \multicolumn{2}{|c|}{$13 \cdot 3$} & \multicolumn{2}{|c|}{$12 \cdot 6$} & \\
\hline Turkish or Moroccan & \multicolumn{2}{|c|}{$5 \cdot 1$} & \multicolumn{2}{|c|}{$20 \cdot 8$} & \\
\hline Surinamese or Antillean & \multicolumn{2}{|c|}{$5 \cdot 3$} & \multicolumn{2}{|c|}{$14 \cdot 2$} & \\
\hline Other non-Western & \multicolumn{2}{|c|}{4.6} & \multicolumn{2}{|c|}{$12 \cdot \overline{3}$} & \\
\hline Maternal education (\%) & & & & & $<0.001$ \\
\hline Primary & \multicolumn{2}{|c|}{2.5} & \multicolumn{2}{|c|}{$14 \cdot 0$} & \\
\hline Secondary & \multicolumn{2}{|c|}{$34 \cdot 0$} & \multicolumn{2}{|c|}{$47 \cdot 3$} & \\
\hline Higher & \multicolumn{2}{|c|}{63.5} & \multicolumn{2}{|c|}{$38 \cdot 7$} & \\
\hline Maternal smoking during pregnancy (\% yes) & \multicolumn{2}{|c|}{$20 \cdot 1$} & & & $<0.001$ \\
\hline Maternal alcohol use during pregnancy ( $\%>1$ glass/week) & & & & & 0.03 \\
\hline Pregnancy planned (\% yes) & & & & & $<0.001$ \\
\hline Breast feeding (\% yes, more than 3 months) & & & & & 0.57 \\
\hline Maternal age in early pregnancy (years) & 31.7 & 4.2 & $29 \cdot 7$ & 5.4 & $<0.001$ \\
\hline Paternal age in early pregnancy (years) & 33.9 & $5 \cdot 0$ & 33.0 & $6 \cdot 6$ & $<0.001$ \\
\hline BMI of mother in early pregnancy $\left(\mathrm{kg} / \mathrm{m}^{2}\right)$ & $24 \cdot 2$ & 4.0 & $24 \cdot 8$ & 4.6 & $<0.001$ \\
\hline GSI score of mother in mid-gestation & & & & & $<0.001$ \\
\hline Median & & & & & \\
\hline $95 \% \mathrm{Cl}$ & & & & & \\
\hline
\end{tabular}

GSI, Global Severity Index of psychiatric symptoms.

${ }^{*} P$ values are derived from independent $t$ tests for continuous normally distributed variables, Mann-Whitney $U$ tests for continuous non-normally distributed variables or $\chi^{2}$ tests for categorical variables.

$19.0 \mathrm{nmol} / \mathrm{l}$. This yielded an OR of 1.7 (95\% CI $0.7,4 \cdot 1$; $P=0.2)$ for total problems in 18-month-old children of mothers who had a low serum folate level compared with children of mothers who had a high serum folate level in early pregnancy. Second, we excluded mothers who reported multivitamin preparation use during the first trimester ( $n$ 1274) and reran the analyses. The adjusted OR for total problems at 18 months of age was $1.34(95 \%$ CI $1.0,1 \cdot 8$; $P=0.04)$ in children of mothers who did not use any folic acid-containing supplements compared with children of mothers who used folic acid-only supplements.

Table 4 shows the association between folic acid use during the first trimester and the broadband and syndrome scales of the CBCL, after adjustment for maternal characteristics. The analyses in Table 4 were, like model 2 in Table 3, adjusted for variables that confound the association between folic acid supplement use and behavioural problems. Since gestational age at birth, birth weight and head growth parameters in late pregnancy did not mediate the associations, they were excluded from the analyses. Children of mothers who did not use folic acid supplements had a higher risk of internalising problems (OR 1.7; $95 \%$ CI $1.2,2.2$ ) and of externalising problems (OR 1.5; $95 \%$ CI 1.2, 1.8). When we stratified our sample on the basis of national origin, all OR for externalising and internalising problems were above 1 .
In the Dutch group ( $n$ 2662), the OR for externalising problems in children of mothers who did not use folic acid supplements was 1.8 (95\% CI 1.4, 2.5; $P<0.001)$.

Maternal folic acid use was significantly related to three syndrome scales (Table 4). The odds for showing withdrawn behaviour were $48 \%$ higher when mothers did not use folic acid supplements in early pregnancy. Second, use of folic acid supplements was related to a lower risk of attention problems and aggressive behaviour. Mothers who started folic acid supplement use after 10 weeks of pregnancy ( $n$ 507) had no lower risk of behavioural problems on any of the subscales compared with mothers who never used folic acid supplements during pregnancy ( $n$ 640) (data not shown).

\section{Discussion}

The present study showed that children of mothers who used folic acid supplements during early pregnancy had a significantly lower risk of behavioural problems, compared with children of mothers who did not use folic acid supplementation.

Apart from the preventive effects of folic acid on neural tube defects ${ }^{(2,3)}$, several lines of research suggest that folates during early pregnancy are important for fetal growth and development. Experimental studies in animals showed the negative effects of prenatal folate deficiency on neural 
Table 2. Subject characteristics according to behavioural information at the age of 18 months (Mean values and standard deviations, medians and $95 \%$ confidence intervals or percentages)

\begin{tabular}{|c|c|c|c|c|c|}
\hline & \multicolumn{2}{|c|}{$\begin{array}{c}\text { Children without } \\
\text { behavioural problems } \\
(n \text { 3513) }\end{array}$} & \multicolumn{2}{|c|}{$\begin{array}{c}\text { Children with } \\
\text { behavioural problems } \\
(n \text { 701) }\end{array}$} & \multirow[b]{2}{*}{$P^{\star}$} \\
\hline & Mean & SD & Mean & SD & \\
\hline Male (\%) & \multicolumn{2}{|c|}{$48 \cdot 4$} & \multicolumn{2}{|c|}{$52 \cdot 8$} & 0.04 \\
\hline Age of the child at measurement (months) & $18 \cdot 4$ & $1 \cdot 1$ & $18 \cdot 5$ & $1 \cdot 1$ & 0.01 \\
\hline Gestational age at birth (weeks) & & & & & 0.26 \\
\hline Median & \multirow{2}{*}{\multicolumn{2}{|c|}{$\begin{array}{c}40 \cdot 1 \\
35.7 \quad 42.4\end{array}$}} & \multicolumn{2}{|c|}{$40 \cdot 0$} & \\
\hline $95 \% \mathrm{Cl}$ & & & \multirow{2}{*}{\multicolumn{2}{|c|}{$\begin{array}{c}35 \cdot 2,42 \cdot 6 \\
6 \cdot 4\end{array}$}} & \\
\hline Preterm birth < 37 weeks (\%) & \multicolumn{2}{|c|}{4.6} & & & 0.04 \\
\hline Birth weight $(\mathrm{g})$ & 3474 & 541 & 3406 & 575 & 0.004 \\
\hline Low birth weight < $2500 \mathrm{~g}(\%)$ & \multicolumn{2}{|c|}{3.9} & \multicolumn{2}{|c|}{5.4} & 0.07 \\
\hline Head circumference in late pregnancy (mm) & $286 \cdot 1$ & $12 \cdot 0$ & $285 \cdot 1$ & $12 \cdot 2$ & 0.06 \\
\hline Transcerebellar diameter in late pregnancy $(\mathrm{mm})$ & 37.9 & 2.4 & 37.7 & 2.4 & 0.09 \\
\hline Atrial width of lateral ventricle in late pregnancy $(\mathrm{mm})$ & $5 \cdot 0$ & $1 \cdot 7$ & 4.9 & 1.7 & 0.38 \\
\hline National origin (\%) & & & & & $<0.001$ \\
\hline Dutch & \multicolumn{2}{|c|}{$66 \cdot 8$} & \multicolumn{2}{|c|}{$45 \cdot 2$} & \\
\hline Other Western & \multicolumn{2}{|c|}{$12 \cdot 9$} & \multicolumn{2}{|c|}{$13 \cdot 8$} & \\
\hline Turkish or Moroccan & \multicolumn{2}{|c|}{$7 \cdot 8$} & \multicolumn{2}{|c|}{$17 \cdot 1$} & \\
\hline Surinamese or Antillean & \multicolumn{2}{|c|}{$7 \cdot 0$} & \multicolumn{2}{|c|}{$11 \cdot 3$} & \\
\hline Other non-Western & \multicolumn{2}{|c|}{$5 \cdot 5$} & \multicolumn{2}{|c|}{$12 \cdot 6$} & \\
\hline Maternal education (\%) & & & & & $<0.001$ \\
\hline Primary & \multicolumn{2}{|c|}{$4 \cdot 2$} & \multicolumn{2}{|c|}{$12 \cdot 4$} & \\
\hline Secondary & \multicolumn{2}{|c|}{$36 \cdot 4$} & \multicolumn{2}{|c|}{43.6} & \\
\hline Higher & \multicolumn{2}{|c|}{$59 \cdot 4$} & \multicolumn{2}{|c|}{43.9} & \\
\hline Maternal smoking during pregnancy (\% yes) & \multicolumn{2}{|c|}{$21 \cdot 2$} & \multicolumn{2}{|c|}{$25 \cdot 4$} & 0.01 \\
\hline Maternal alcohol use during pregnancy $(\%>1$ glass/week $)$ & \multicolumn{2}{|c|}{$26 \cdot 0$} & & & 0.004 \\
\hline Pregnancy planned (\% yes) & & & & & $<0.001$ \\
\hline Breast feeding (\% yes, more than 3 months) & & & & & 0.24 \\
\hline Maternal age in early pregnancy (years) & 31.4 & 4.4 & 29.8 & 5.4 & $<0.001$ \\
\hline Paternal age in early pregnancy (years) & $33 \cdot 8$ & $5 \cdot 3$ & $32 \cdot 9$ & $6 \cdot 1$ & 0.001 \\
\hline BMI of mother in early pregnancy $\left(\mathrm{kg} / \mathrm{m}^{2}\right)$ & $24 \cdot 2$ & $4 \cdot 1$ & $24 \cdot 8$ & 4.5 & 0.003 \\
\hline GSI score of mother in mid-gestation & & & & & $<0.001$ \\
\hline Median & 0.12 & & 0.25 & & \\
\hline $95 \% \mathrm{Cl}$ & $0,1 \cdot 02$ & & $0,1 \cdot 78$ & & \\
\hline
\end{tabular}

GSI, Global Severity Index of psychiatric symptoms.

${ }^{*} P$ values are derived from independent $t$ tests for continuous normally distributed variables, Mann-Whitney $U$ tests for continuous non-normally distributed variables or $\chi^{2}$ tests for categorical variables.

development $^{(5)}$ and virtually all inborn errors of folate metabolism are associated with mental retardation ${ }^{(20)}$. One of the very few studies on human neurodevelopment found no significant association between folate nutritional status of pregnant women and mental and psychomotor development in their children at 5 years of age ${ }^{(11)}$. The discrepancy between our findings and these results from Tamura et al. may be explained by the small number of mothers with low plasma folate, and the follow-up of a non-population-based sample of educationally and environmentally deprived women in their study ${ }^{(11)}$. Furthermore, the inconsistency may be explained by differences in measurement of folate intake and neurodevelopmental outcome.

Several potential mechanisms may explain the association between folic acid use by pregnant women and behavioural problems in the offspring. First, folates may directly affect the developing central nervous system. It is still not clear whether neural tube defects arise from folate deficiency, or whether folate supplementation overcomes an intrinsic defect in folate metabolism ${ }^{(1,21)}$. Folate is important in neurogenesis, in cell growth and proliferation, and in myelination. Furthermore, folate has been linked to the synthesis of catecholamine neurotransmitters and $\operatorname{serotonin}^{(22)}$.
Additionally, animal studies provided information on the requirements for folate intake later in fetal gestation. Craciunescu et al. ${ }^{(5)}$ reported that dietary folic acid availability affects mouse brain development long after neural tube closure by influencing cell mitosis and apoptosis in the brain. Although we did not find significant mediation by the size of the cerebrum, cerebellum or ventricles, our findings may be explained by subtle changes in neuronal and glial growth and proliferation or on the level of neurotransmitters.

Second, indirect effects on fetal growth may explain the effects of prenatal folate deficiency on behavioural problems in young children. Iyengar \& Rajalakshmi ${ }^{(4)}$ and Scholl \& Johnson $^{(23)}$ described higher birth weights in children of mothers who used folic acid supplementation during pregnancy. The effects of folate on placental vasculopathy ${ }^{(24)}$ or on DNA methylation of growth hormones in fetal tissues serve as possible mechanisms. A lower birth weight has, in turn, been related to behavioural and emotional problems ${ }^{(25)}$. However, the present results do not underscore the hypothesis that birth weight mediates the effects of folate deficiency in prenatal life on behavioural development.

Third, an alternative indirect effect of folate deficiency may be de novo mutations or epigenetic dysregulation of genes. 
Table 3. Maternal folic acid use and child behavioural problems (total problems score) ( $n 4214$ subjects in the analyses) (Odds ratios and $95 \%$ confidence intervals)

\begin{tabular}{|c|c|c|c|c|c|c|c|c|}
\hline & \multicolumn{2}{|c|}{ Model $1^{*}$} & \multicolumn{2}{|c|}{ Model 2† } & \multicolumn{2}{|c|}{ Model $3 \ddagger$} & \multicolumn{2}{|c|}{ Model $4 \S$} \\
\hline & OR & $95 \% \mathrm{Cl}$ & OR & $95 \% \mathrm{Cl}$ & OR & $95 \% \mathrm{Cl}$ & OR & $95 \% \mathrm{Cl}$ \\
\hline \multicolumn{9}{|l|}{ Folic acid use } \\
\hline Supplement use during the first 10 weeks of pregnancy & \multicolumn{2}{|c|}{ Reference } & \multicolumn{2}{|c|}{ Reference } & \multicolumn{2}{|c|}{ Reference } & \multicolumn{2}{|c|}{ Reference } \\
\hline $\begin{array}{l}\text { No supplement use } \\
P\end{array}$ & $2 \cdot 78$ & $\begin{aligned} & 2.24,3.44 \\
< & 0.001\end{aligned}$ & 1.44 & $\begin{array}{l}1 \cdot 12,1 \cdot 86 \\
0.005\end{array}$ & 1.44 & $\begin{array}{l}1 \cdot 12,1 \cdot 86 \\
0.005\end{array}$ & 1.43 & $\begin{array}{l}1 \cdot 11,1.85 \\
0.005\end{array}$ \\
\hline $\begin{array}{l}\text { Maternal age (per year) } \\
\quad P\end{array}$ & & - & 0.96 & $\begin{array}{l}0.94,0.98 \\
0.001\end{array}$ & 0.96 & $\begin{array}{l}0.94,0.98 \\
0.001\end{array}$ & 0.96 & $\begin{array}{l}0.94,0.98 \\
0.001\end{array}$ \\
\hline \multicolumn{9}{|l|}{ National origin } \\
\hline Dutch & & - & \multicolumn{2}{|c|}{ Reference } & \multicolumn{2}{|c|}{ Reference } & \multicolumn{2}{|c|}{ Reference } \\
\hline Other Western & & - & 1.96 & $1.40,2.79$ & 1.97 & $1 \cdot 40,2 \cdot 78$ & 1.97 & $1 \cdot 40,2 \cdot 78$ \\
\hline Turkish or Moroccan & & - & 3.07 & $2 \cdot 15,4.35$ & $3 \cdot 11$ & $2 \cdot 19,4.42$ & 3.09 & $2 \cdot 18,4 \cdot 40$ \\
\hline Surinamese or Dutch Antillean & & - & $2 \cdot 30$ & $1.59,3.41$ & $2 \cdot 27$ & $1.54,3.36$ & $2 \cdot 31$ & $1.56,3.40$ \\
\hline Other non-Western & & - & 3.49 & $2.41,5.04$ & 3.48 & $2 \cdot 40,5 \cdot 04$ & 3.49 & $2 \cdot 41,5.06$ \\
\hline$P \|$ & & & & $<0.001$ & & $<0.001$ & & $<0.001$ \\
\hline \multicolumn{9}{|l|}{ Educational level } \\
\hline Primary & & - & 1.96 & $1.31,2.94$ & 1.96 & $1.31,2.94$ & 1.96 & $1.31,2.94$ \\
\hline Secondary & & - & $1 \cdot 15$ & $0.88,1.51$ & $1 \cdot 13$ & $0.86,1.48$ & 1.14 & $0.87,1.50$ \\
\hline Higher & & - & \multirow{2}{*}{\multicolumn{2}{|c|}{$\begin{array}{c}\text { Reference } \\
0.01\end{array}$}} & \multicolumn{2}{|c|}{ Reference } & \multicolumn{2}{|c|}{ Reference } \\
\hline$P \|$ & & & & & & 0.01 & & 0.01 \\
\hline $\begin{array}{l}\text { Maternal psychopathology (per SD) } \\
\quad P\end{array}$ & & - & $1 \cdot 32$ & $\begin{aligned} & 1.22,1.44 \\
< & 0.001\end{aligned}$ & 1.32 & $\begin{aligned} & 1.22,1.44 \\
< & 0.001\end{aligned}$ & $1 \cdot 32$ & $\begin{array}{l}1.22,1.44 \\
<0.001\end{array}$ \\
\hline $\begin{array}{l}\text { Birth weight (per SD) } \\
\quad P\end{array}$ & & - & & - & 0.98 & $\begin{array}{l}0.87,1 \cdot 11 \\
0 \cdot 78\end{array}$ & & - \\
\hline $\begin{array}{l}\text { Gestational age at birth (per week) } \\
P\end{array}$ & & - & & - & 0.94 & $\begin{array}{l}0.89,1.00 \\
0.04\end{array}$ & & - \\
\hline $\begin{array}{l}\text { Head circumference in late pregnancy (per SD) } \\
\quad P\end{array}$ & & - & & - & & - & 0.96 & $\begin{array}{l}0.85,1.08 \\
0.49\end{array}$ \\
\hline
\end{tabular}

* Model 1 = adjusted for age and sex.

† Model 2 = model 1 , additionally adjusted for maternal characteristics (i.e. maternal age, national origin, educational level and psychopathology).

$\ddagger$ Model $3=$ model 2 , additionally adjusted for birth weight and gestational age at birth.

$\S$ Model $4=$ model 2 , additionally adjusted for head circumference in late pregnancy.

\| Overall $P$ value for categorical variables.

McClellan et al. hypothesised that the higher incidence of schizophrenia in offspring exposed to prenatal famine may be due to de novo mutations or epigenetic modifications ${ }^{(26)}$. Animal studies showed that expression of genes could be modified by maternal folate supplementation. In the agouti mouse, for example, dietary supplementation was highly correlated with the degree of methylation, which in turn predicted coat colour and a higher risk of obesity, cancer, diabetes and short life $\operatorname{span}^{(27)}$.

Fourth, the findings of the present study may be explained by the epiphenomenona of folic acid use during pregnancy. The present study showed that folic acid supplementation is highly related to socio-economic class and lifestyle determinants. Maternal characteristics, such as age, educational level, national origin and psychopathology, were important confounders in the reported association, although they did not completely erode the effect of prenatal folates on child behaviour. Nevertheless, residual confounding remains an issue in all observational studies. For example, intake of other macro- and micronutrients, as well as genetic polymorphisms that could affect folate metabolism, may well serve as additional confounding factors. A relatively large proportion of mothers reporting folic acid supplement use also used multivitamins during early pregnancy. We tried to overcome this problem of misclassification by excluding mothers who reported multivitamin use, but residual confounding may still affect the present results. Although the information on maternal dietary intake as well as genetic information in both mothers and children were collected within the Generation R Study, the data are not ready for analyses.

Other methodological considerations include selection and information bias. Children and mothers without information on behavioural outcome differed from families with behavioural information on both the determinant (folic acid use during pregnancy) and several confounding factors. The use of self-reports of folic acid supplement use during pregnancy is a second potential limitation. However, validation studies $^{(28)}$ showed that self-reported intake of folic acid supplements strongly correlated with serum folates, which was replicated in a small subsample of the present study. Moreover, we prospectively assessed supplement use in early pregnancy, which makes recall bias unlikely. Another source of possible reporter bias is the use of a mother report to assess behavioural problems, as well as the use of self-reported information to assess maternal psychopathology. This important confounding variable was measured with the Brief Symptom Inventory, which is a validated self-report on symptoms of psychopathology, but does not provide information on Diagnostic and Statistical Manual of Mental Disorders, 4th edition (DSM-IV) diagnoses.

In conclusion, in our data, failure to use folic acid supplements during early pregnancy is associated with child behavioural and emotional problems later in life. Future studies are needed to replicate the association and to 
Table 4. Maternal folic acid use supplementation during embryogenesis and child behavioural problems ( $n 4214$ subjects in the analyses)*

(Odds ratios and $95 \%$ confidence intervals)

\begin{tabular}{|c|c|c|c|}
\hline $\begin{array}{l}\text { Child Behaviour Checklist broadband } \\
\text { scales }\end{array}$ & OR & $95 \% \mathrm{Cl}$ & $P$ \\
\hline Internalising & \multirow{2}{*}{\multicolumn{2}{|c|}{ Reference }} & 0.001 \\
\hline Folic acid supplement use & & & \\
\hline No use & 1.65 & $1 \cdot 24,2 \cdot 19$ & \\
\hline \multicolumn{4}{|l|}{ Internalising syndrome scales } \\
\hline Emotionally reactive & & & 0.33 \\
\hline Folic acid supplement use & \multicolumn{2}{|c|}{ Reference } & \\
\hline No use & 1.26 & $0.79,1.99$ & \\
\hline Anxious/depressed & & & 0.18 \\
\hline Folic acid supplement use & \multicolumn{2}{|c|}{ Reference } & \\
\hline No use & 1.33 & $0.88,2.01$ & \\
\hline Somatic complaints & & & $0 \cdot 12$ \\
\hline Folic acid supplement use & \multicolumn{2}{|c|}{ Reference } & \\
\hline No use & 1.32 & $0.93,1.86$ & \\
\hline Withdrawn & & & 0.007 \\
\hline Folic acid supplement use & \multicolumn{2}{|c|}{ Reference } & \\
\hline No use & 1.48 & $1.11,1.96$ & \\
\hline Externalising & & & 0.001 \\
\hline Folic acid supplement use & \multicolumn{2}{|c|}{ Reference } & \\
\hline No use & 1.45 & $1.17,1.80$ & \\
\hline \multicolumn{4}{|l|}{ Externalising syndrome scales } \\
\hline Attention problems & & & 0.03 \\
\hline Folic acid supplement use & \multicolumn{2}{|c|}{ Reference } & \\
\hline No use & 1.30 & $1.03,1.64$ & \\
\hline Aggressive behaviour & & & 0.03 \\
\hline Folic acid supplement use & \multicolumn{2}{|c|}{ Reference } & \\
\hline No use & 1.51 & $1.04,2.19$ & \\
\hline
\end{tabular}

investigate whether prenatal folate deficiency also predicts neurodevelopmental disorders, such as attention-deficit hyperactivity disorder and schizophrenia. Despite the current recommendations of adequate folic acid use, still many pregnant women, and in particular those with other vulnerability markers for physical and mental health, do not use folic acid supplements preconceptionally or as soon as pregnancy is known. Development and application of preconceptional health educational programmes to improve intake of folic acid and other health-related behaviours is therefore highly recommended.

\section{Acknowledgements}

The Generation $\mathrm{R}$ Study is conducted by the Erasmus Medical Center (Rotterdam, The Netherlands) in close collaboration with the Faculty of Social Sciences of the Erasmus University Rotterdam, the Municipal Health Service Rotterdam area, the Rotterdam Homecare Foundation and the Stichting Trombosedienst \& Artsenlaboratorium Rijnmond (STAR), Rotterdam. We gratefully acknowledge the contribution of general practitioners, hospitals, midwives and pharmacies in Rotterdam. The first phase of Generation $\mathrm{R}$ was supported by the Erasmus Medical Center, the Erasmus University (Rotterdam, The Netherlands), and the Netherlands Organization for Health Research and Development (ZonMw). The research leading to these results has received funding from The Netherlands Organization for
Health Research and Development (ZonMw, 'Geestkracht' programme 10.000.1003) and the European Community's 7th Framework Programme (FP7/2008-2013) under grant agreement no. 212652 (NUTRIMENTHE Project 'The Effect of Diet on the Mental Performance of Children').

A. H. is guarantor and Jaddoe project leader of the Generation R Study. H. T., F. C. V., E. A. P. S. and S. J. R. conceived of and designed the present study. E. A. P. S. supervised and coordinated the collection of information on folic acid supplements. S. J. R. and H. T. analysed and interpreted the data. All authors contributed to drafting or revising the manuscript and read and approved the final version.

The authors declare that there are no conflicts of interest.

\section{References}

1. Bailey LB \& Gregory JF III (1999) Folate metabolism and requirements. J Nutr 129, 779-782.

2. Anonymous (1991) Prevention of neural tube defects: results of the Medical Research Council Vitamin Study. MRC Vitamin Study Research Group. Lancet 338, 131-137.

3. Lumley J, Watson L, Watson M, et al. (2001) Periconceptional supplementation with folate and/or multivitamins for preventing neural tube defects. The Cochrane Database of Systematic Reviews 2001, issue 3, CD001056. http://www. mrw.interscience.wiley.com/cochrane/clsysrev/articles/ CD001056/frame.html

4. Iyengar L \& Rajalakshmi K (1975) Effect of folic acid supplement on birth weights of infants. Am J Obstet Gynecol 122, 332-336.

5. Craciunescu CN, Brown EC, Mar MH, et al. (2004) Folic acid deficiency during late gestation decreases progenitor cell proliferation and increases apoptosis in fetal mouse brain. J Nutr 134, 162-166.

6. Xiao S, Hansen DK, Horsley ET, et al. (2005) Maternal folate deficiency results in selective upregulation of folate receptors and heterogeneous nuclear ribonucleoprotein-E1 associated with multiple subtle aberrations in fetal tissues. Birth Defects Res A Clin Mol Teratol 73, 6-28.

7. Zammit S, Lewis S, Gunnell D, et al. (2007) Schizophrenia and neural tube defects: comparisons from an epidemiological perspective. Schizophr Bull 33, 853-858.

8. Brown AS, Bottiglieri T, Schaefer CA, et al. (2007) Elevated prenatal homocysteine levels as a risk factor for schizophrenia. Arch Gen Psychiatry 64, 31-39.

9. Erbe RE (1975) Inborn errors of folate metabolism (first of two parts). N Engl J Med 293, 753-757.

10. Erbe RW (1975) Inborn errors of folate metabolism (second of two parts). N Engl J Med 293, 807-812.

11. Tamura T, Goldenberg RL, Chapman VR, et al. (2005) Folate status of mothers during pregnancy and mental and psychomotor development of their children at five years of age. Pediatrics 116, 703-708.

12. Jaddoe VW, Mackenbach JP, Moll HA, et al. (2006) The Generation R Study: design and cohort profile. Eur $J$ Epidemiol 21, 475-484.

13. Achenbach TM \& Rescorla LA (2000) Manual for the ASEBA Preschool Forms and Profiles. Burlington, VT: University of Vermont, Research Center for Children, Youth, and Families.

14. Tick NT, van der Ende J, Koot HM, et al. (2007) 14-Year changes in emotional and behavioural problems of very young Dutch children. J Am Acad Child Adolesc Psychiatry 46, 1333-1340.

15. Reichenberg A, Gross R, Weiser M, et al. (2006) Advancing paternal age and autism. Arch Gen Psychiatry 63, 1026-1032. 
16. Malaspina D, Harlap S, Fennig S, et al. (2001) Advancing paternal age and the risk of schizophrenia. Arch Gen Psychiatry 58, 361-367.

17. de Beurs E (2004) Brief Symptom Inventory. Leiden, The Netherlands: Pits Publishers.

18. Derogatis LR \& Melisaratos N (1983) The Brief Symptom Inventory: an introductory report. Psychol Med 13, 595-605.

19. Timmermans S, Jaddoe VW, Mackenbach JP, et al. (2008) Determinants of folic acid use in early pregnancy in a multi-ethnic urban population in the Netherlands: The Generation R Study. Prev Med (Epublication ahead of print version 2 July 2008).

20. Greenblatt JM, Huffman LC \& Reiss AL (1994) Folic acid in neurodevelopment and child psychiatry. Prog Neuropsychopharmacol Biol Psychiatry 18, 647-660.

21. Brown AS, Susser ES, Butler PD, et al. (1996) Neurobiological plausibility of prenatal nutritional deprivation as a risk factor for schizophrenia. J Nerv Ment Dis 184, 71-85.
22. Fernstrom JD (2000) Can nutrient supplements modify brain function? Am J Clin Nutr 71, Suppl. 6, 1669S-1675S.

23. Scholl TO \& Johnson WG (2000) Folic acid: influence on the outcome of pregnancy. Am J Clin Nutr 71, Suppl. 5, 1295S-1303S

24. Tamura T \& Picciano MF (2006) Folate and human reproduction. Am J Clin Nutr 83, 993-1016.

25. Kelly YJ, Nazroo JY, McMunn A, et al. (2001) Birthweight and behavioural problems in children: a modifiable effect? Int J Epidemiol 30, 88-94.

26. McClellan JM, Susser E \& King M (2006) Maternal famine, de novo mutations, and schizophrenia. JAMA 296, 582-584.

27. Wolff GL, Kodell RL, Moore SR, et al. (1998) Maternal epigenetics and methyl supplements affect agouti gene expression in Avy/a mice. FASEB J 12, 949-957.

28. Brantsaeter AL, Haugen M, Hagve TA, et al. (2007) Selfreported dietary supplement use is confirmed by biological markers in the Norwegian Mother and Child Cohort Study (MoBa). Ann Nutr Metab 51, 146-154. 